\title{
Madelung's Deformity of the Wrist-Current Concepts and Future Directions
}

\author{
Satish Babu, BSc (Hons), MBBS, MRCS ${ }^{1}$ Joseph Turner, BMedSci (Hons), MBBS, MRCS² \\ Sheena Seewoonarain, MRCS ${ }^{3}$ Sanjay Chougule, FRCS (Tr \& Orth $)^{4}$
}

1 Frimley Park Hospital, Portsmouth Road, Frimley, United Kingdom

2 East Surrey Hospital, Canada Avenue, Redhill, United Kingdom

${ }^{3}$ St. Richard's Hospital, Spitalfield Lane, Chichester, United Kingdom

${ }^{4}$ East Surrey Hospital, Canada Avenue, Redhill, United Kingdom

Address for correspondence Joseph Turner, BMedSci (Hons), MBBS, MRCS, East Surrey Hospital, Canada Avenue, Redhill, RH1 5RH, United Kingdom (e-mail: josephturner@nhs.net).

J Wrist Surg 2019;8:176-179.

\begin{abstract}
Keywords

- Madelung

- wrist

- DRUJ

- deformity

- congenital

Madelung's deformity of the wrist arises from premature closure of the medial and volar aspect of the distal radial physis. True Madelung deformities reveal the presence of a "Vickers" ligament which is a short, volar, radioulnar ligament. Clinically, patients report increasing deformity, pain, and poor range of motion. Radiological features include increased radial inclination, volar tilt of the distal radius, and a positive ulnar variance. Surgical intervention usually comprises either a "Vickers" ligament release and distal radius physiolysis or a radial dome osteotomy. In future, EOS Imaging could aid diagnosis by providing more detailed images of the deformity while minimizing radiation exposure. Furthermore, three-dimensional printing and computer-navigated deformity correction could revolutionize management by facilitating simulation training, expediting surgery, and reducing intraoperative error.
\end{abstract}

Madelung's deformity of the wrist was first officially described by Otto Madelung in $1878 .^{1}$ This deformity arises in adolescents aged 8 to 14 and is often bilateral. ${ }^{1,2}$ It is more common in females showing a $4: 1$ predominance and represents less than $2 \%$ of all pediatric hand deformities. ${ }^{3,4}$

The deformity stems from premature closure of the medial volar aspect of the distal radial physis. ${ }^{5}$ This subsequently leads to an increased radial inclination and volar tilt of the distal radius, proximal migration of the lunate with triangulation of the carpus, and the characteristic dorsal displacement of the ulna. $^{3}$ Secondary abnormalities of the ulna, carpus, articular cartilage, and tendons can occur as a result of this abnormal growth pattern and deformity. ${ }^{3}$ Unfortunately, despite its documentation in the literature, Madelung's deformity is poorly understood among clinicians and often misdiagnosed.

\section{Etiology}

The etiology of Madelung's deformity remains unclear, but there is a genetic association. Congenital Madelung deformities are associated with a mutation or absence of the short

received

December 22, 2018

accepted

March 4, 2019

published online

April 22, 2019 stature homeobox (SHOX) gene in the pseudoautosomal region of sex chromosomes. ${ }^{5,6}$

The same genetic deficiencies are found in patients with Leri Weill dyschondrosteosis, a condition associated with Madelung deformities. ${ }^{2}$ These deformities are also linked with other genetic disorders including Turners syndrome. ${ }^{7}$ Cases associated with abnormal SHOX genes are often bilateral and symmetrical. Idiopathic cases can also occur, although these are usually unilateral.,8

Apart from true Madelung deformities, Madelung-like deformities are seen in conditions including multiple hereditary exostoses and Ollier disease. ${ }^{2,3}$ Indeed, any condition causing injury or arrest of the medial volar radial physis can produce this type of deformity, a phenomenon often seen in gymnasts due to repetitive trauma. ${ }^{9}$

The key to distinguishing between a true Madelung's deformity and Madelung-like deformities is the presence of a "Vickers" ligament. This is seen in true Madelung deformities and represents an abnormally short, volar, radioulnar ligament. ${ }^{10}$ Usually this ligament originates from the radial epiphysis but in this state comes from the medial radial metaphysis.
Copyright $\odot 2019$ by Thieme Medical Publishers, Inc., 333 Seventh Avenue, New York, NY 10001, USA. Tel: +1(212) 584-4662.
DOI https://doi.org/ $10.1055 / \mathrm{s}-0039-1685488$. ISSN 2163-3916. 
The "Vickers" ligament restricts medial and volar growth of the radius by exerting a compressive effect on the volarulnar physis. $^{10,11}$ However, the remainder of the physis demonstrates normal growth resulting in the characteristic features of increased radial inclination and volar tilt. ${ }^{2}$

Simultaneous unaffected growth of the ulna results in a positive ulnar variance, and an abnormal formation of the distal radioulnar joint (DRUJ) leads to dorsal dislocation of the distal ulna. ${ }^{2}$ Despite this distinction between true Madelung's and Madelung-type deformities, the clinical presentation, investigation, and management are very similar between the two.

\section{Clinical Presentation}

Patients with these deformities have a range of presentations dependent on their age and severity of deformity. Initially individuals are asymptomatic and the most common presentation is a progressive clinical deformity. Patients report a prominent distal ulna as a result of dorsal subluxation, often termed a "bayonet" deformity. ${ }^{3}$ Interestingly, patients are usually unaware of the distal radial abnormality or carpal subluxation.

Reduced range of motion is a common feature with forearm rotation often compromised. Loss of wrist extension is common and proportional to the degree of sagittal deformity. ${ }^{2}$ Unsurprisingly, radiocarpal pain is frequently found particularly on wrist extension as is pain from ulnar abutment, although this seems more severe in milder deformities prior to DRUJ dislocation. ${ }^{2}$ Individuals may also report reduced grip strength and difficulty executing normal daily activities. 5,12

\section{Radiological Features}

$\mathrm{X}$-ray investigation reveals certain pathognomonic findings. Anteroposterior wrist views reveal a radiolucent flameshaped notch at the medial radial metaphysis produced by the "Vickers" ligament. ${ }^{2}$ They also demonstrate an increased radial inclination, proximal, and volar migration of the lunate with triangulation of the carpus and a positive ulnar variance. $^{3,13}$ On lateral X-rays, classic findings include an increased radial tilt, volar subluxation of the carpus, and dorsal subluxation of the distal ulna. This "subluxation" may seem exaggerated due to the volar radial tilt. ${ }^{3}$ Other features of chronicity may be found including an irregular ulnar head due to the positive variance (-Fig. $\mathbf{1}$ ).

McCarroll et al go further by suggesting X-ray parameters to aid diagnosis. ${ }^{13}$ These include a minimum of $33^{\circ}$ of ulnar tilt, $4 \mathrm{~mm}$ of lunate subsidence, $20 \mathrm{~mm}$ of palmar carpal displacement, and a lunate fossa angle of at least $40^{\circ} .{ }^{13,14}$ However, the authors themselves acknowledge only the first two of the above parameters have excellent reliability and reproducibility.

Magnetic resonance imaging (MRI) is not necessary but can be helpful. This would allow identification of the "Vickers" ligament, degree of physeal disease, and a three-dimensional (3D) view of the deformity. ${ }^{11}$ Additionally, MRI may reveal an anomalous volar radiotriquetral ligament and a thick, obliquely oriented triangular fibrocartilage complex (TFCC) due to the radial deformity. ${ }^{3,11}$ Secondary features including cartilaginous and extensor tendon tears can also be visualized, which, if chronic, may present with minimal symptoms. Computed tomography (CT) scanning serves a purpose when planning surgical correction.

\section{Management}

The large spectrum of Madelung's deformity necessitates a multivariate approach to management. After initial diagnosis, a potential genetic etiology must be considered and genetic counselling must be offered to the patient and family.

During management, the patient's skeletal maturity, severity of deformity, and symptoms should be evaluated.
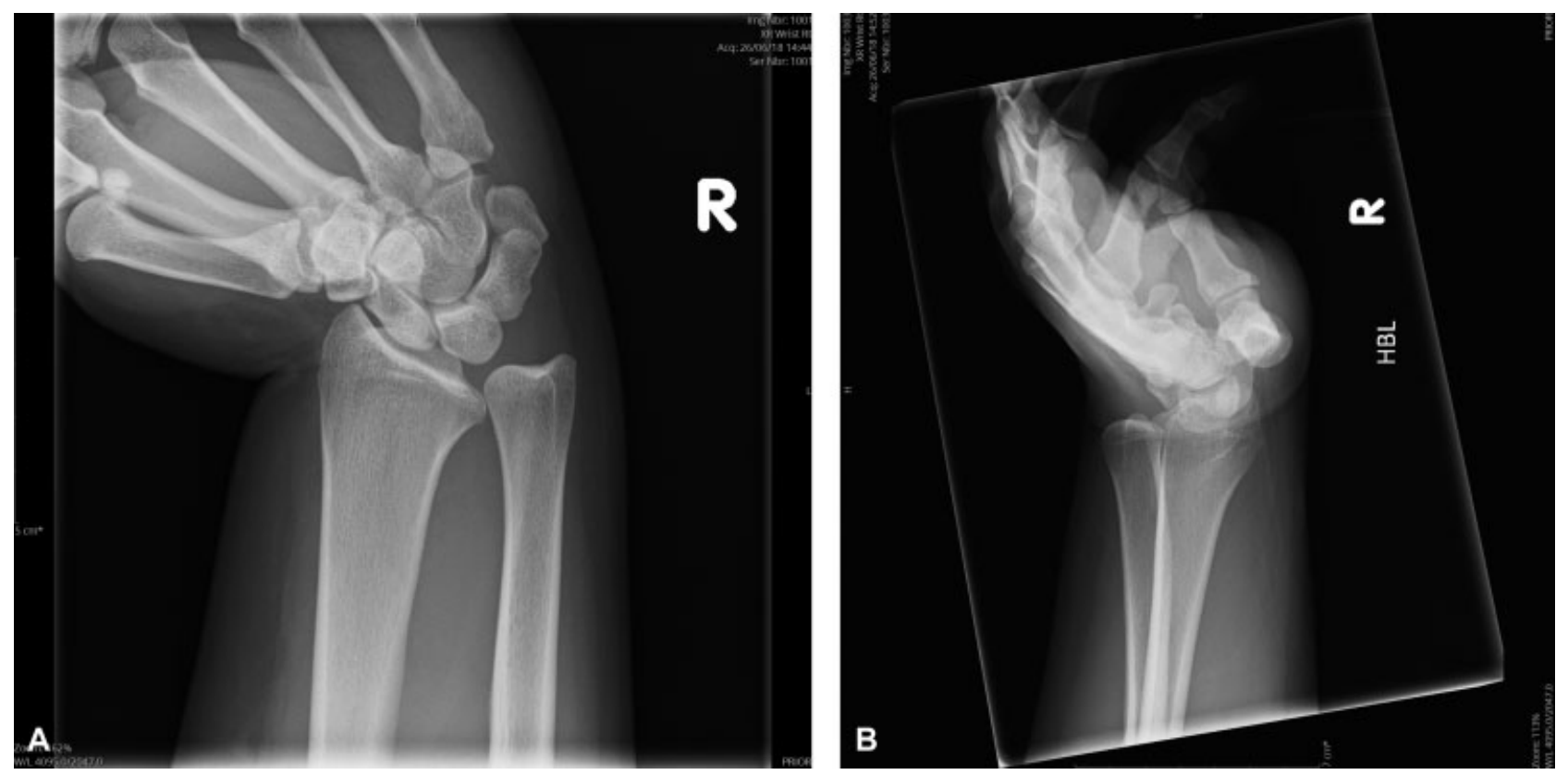

Fig. 1 (A) Anteroposterior and (B) lateral X-rays of our patient showing increased radial inclination, volar tilt, volar lunate migration with triangulation of the carpus, positive ulnar variance, and dorsal subluxation of the distal ulna. 
Mild deformities in patients approaching skeletal maturity may be confidently left alone. Mild deformities in younger individuals may warrant active surveillance with serial Xrays. Furthermore, those who decline surgery may benefit from custom orthoses to alleviate symptoms.

When deformity or symptoms are severe, surgery can restore alignment. Madelung's deformity is associated with abnormalities in many bones and articular surfaces. As such multiple options exist which target specific components of the deformity. Historically, the most favored operative solution is a "Vickers" ligament release combined with a physiolysis, often referred to as the Langenskiöld procedure. ${ }^{15}$ During this operation, the "Vickers" ligament is released from its proximal attachment to the radial metaphysis. Physeal bars or bony bridges are resected and the physis is interposed with fat.

This can be further combined with a "guided growth" tactic. Staples can be placed across the unaffected physis to limit growth and correct the deformity. ${ }^{2}$ Obviously, this strategy relies on a future growth potential from the patient and is therefore not suitable for advanced deformities or those with little growth left.

Another option for ulnar abutment symptoms is an ulnar shortening osteotomy, which can be performed using a variety of techniques. While this does not correct the underlying problem, it does deal with the common sequelae of a positive ulnar variance and subsequent ulnar-sided wrist pain.

One variation in this, the Sauvé-Kapandji technique, employs a DRUJ arthrodesis and ulnar resection osteotomy proximal to the arthrodesis. ${ }^{16}$ This has the advantage of correcting the dorsal dislocation of the distal ulna which is the most common cosmetic complaint in Madelung's deformity. Furthermore, the proximal site of the osteotomy leaves the ulnar head and ulnocarpal ligaments undisturbed, therefore maintaining the ulnar support of the wrist. ${ }^{16}$ This technique also maintains good forearm rotation. If further ulnar growth potential remains, a distal ulnar epiphysiodesis can be performed in conjunction with this technique.

The option that addresses the deformity most thoroughly is a radial osteotomy. Techniques which have produced improvements in pain and range of motion include reverse wedge osteotomies and lengthening using Ilizarov frames. ${ }^{17,18} \mathrm{How}-$ ever, the current gold standard is a radial dome osteotomy. This allows a more precise, 3D deformity correction which will achieve a better realignment compared with standard techniques. ${ }^{19}$ This dome osteotomy is often combined with a "Vickers" ligament release and physiolysis. One retrospective study has shown that this technique produced long-term improvements in pain and functional outcomes. ${ }^{16}$

\section{Future Directions and Application of Innovations}

In diagnosing Madelung's deformity, clinicians have been reluctant to accept the diagnostic criteria proposed by McCarroll et al. This is suggested to be due to inherent deficiencies in the X-ray images. ${ }^{20} \mathrm{X}$-ray provides a twodimensional picture of a complex, 3D problem and may be insufficient for modern management.
High-resolution CT scanning with 3D reconstruction would allow the generation of highly detailed images of the deformity. However, Madelung's deformity usually affects adolescents who may need frequent, serial imaging. High dose radiation is best avoided in this cohort due to potential systemic side effects.

EOS Imaging could address this problem. This X-ray system provides simultaneous anteroposterior and lateral X-rays while utilizing a low radiation dose. ${ }^{21,22}$ Three-dimensional reconstructions can be generated from the X-rays allowing visualization of bony anatomy, critical angles, and deformities. ${ }^{23}$ Indeed, evidence shows EOS Imaging are as good as CT when assessing femoral and tibial torsion measurements. ${ }^{24}$ EOS Imaging has also been found to be more accurate than CT and conventional radiography when measuring bone length. ${ }^{25}$

Therefore, EOS Imaging could revolutionize the workup of Madelung's deformity patients by providing an extremely accurate, 3D model of their anatomy while limiting X-ray exposure.

Three-dimensional printing would also allow more thorough investigation of Madelung deformities. One advantage is the ability to truly appreciate complex anatomy by coordinating one's visuospatial orientation. Furthermore, seeing the model in true form may highlight previously unrecognized problems. One retrospective study showed preoperative 3D printing provided surgeons with better understanding of anatomy, fracture pattern and was effectively used for preoperative planning. ${ }^{26}$

Three-dimensional printing would also improve the management of Madelung deformities. Three-dimensional printed models would allow simulation training and enable mock operations in a risk-free environment. Indeed, preoperative simulation using 3D printed models has produced shorter operation times, less blood loss, shorter fluoroscopy times, and higher rates of anatomic reduction..$^{27-29}$

EOS Imaging and 3D printing could be used to produce customized intraoperative jigs. These would be templated to the patient's individual deformity and guide the osteotomy intraoperatively. They would no doubt have the same advantages of expedited surgery and limited fluoroscopy while minimizing surgical error.

Computer-navigated deformity correction can also be used in surgical management to produce exact osteotomy cuts and reduce operative time and minimize intraoperative error. Evidence shows computer-navigated pelvic and high tibial osteotomies are significantly more accurate and reproducible than non-navigated cuts. ${ }^{30,31}$

The innovations outlined above have the potential to deliver patient-centered, individualized surgery which will hopefully provide better pain and functional outcomes in patients with Madelung deformities.

\section{Conclusion}

Madelung's deformity represents an umbrella term for a wide spectrum of disease. Deformities can be a true Madelung (identified by a "Vickers" ligament) or Madelung-type. Clinical features include a characteristic deformity and reduced range of motion and pain. Initial treatment may 
involve active surveillance and orthoses. Surgical options depend on the symptoms being addressed but include the Langenskiöld procedure or radial dome osteotomy. In future, EOS Imaging could improve diagnosis by allowing a more accurate appreciation of the anatomy. Furthermore, 3D printing and computer-navigated correction could revolutionize management by allowing simulation training, expediting surgery, and reducing intraoperative error.

\section{Conflict of Interest}

None declared.

\section{References}

1 Arora AS, Chung KC, Otto W. Madelung and the recognition of Madelung's deformity. J Hand Surg Am 2006;31(02):177-182

2 Kozin SH, Zlotolow DA. Madelung deformity. J Hand Surg Am 2015;40(10):2090-2098

3 Ali S, Kaplan S, Kaufman T, Fenerty S, Kozin S, Zlotolow DA. Madelung deformity and Madelung-type deformities: a review of the clinical and radiological characteristics. Pediatr Radiol 2015;45(12):1856-1863

4 Flatt AE. The Care of Congenital Hand Anomalies. St. Louis, MO: Mosby; 1994

5 Ghatan AC, Hanel DP. Madelung deformity. J Am Acad Orthop Surg 2013;21(06):372-382

6 Benito-Sanz S, Thomas NS, Huber C, et al. A novel class of pseudoautosomal region 1 deletions downstream of SHOX is associated with Leri-Weill dyschondrosteosis. Am J Hum Genet 2005;77(04):533-544

7 Clement-Jones M, Schiller S, Rao E, et al. The short stature homeobox gene SHOX is involved in skeletal abnormalities in Turner syndrome. Hum Mol Genet 2000;9(05):695-702

8 Plafki C, Luetke A, Willburger RE, Wittenberg RH, Steffen R. Bilateral Madelung's deformity without signs of dyschondrosteosis within five generations in a European family-case report and review of the literature. Arch Orthop Trauma Surg 2000;120(1-2):114-117

9 Tranmer A, Laub D Jr. Madelung deformity. Eplasty 2016;16:ic34

10 Vickers D, Nielsen G. Madelung deformity: surgical prophylaxis (physiolysis) during the late growth period by resection of the dyschondrosteosis lesion. J Hand Surg [Br] 1992;17(04):401-407

11 Stehling C, Langer M, Nassenstein I, Bachmann R, Heindel W, Vieth V. High resolution 3.0 Tesla MR imaging findings in patients with bilateral Madelung's deformity. Surg Radiol Anat 2009;31(07):551-7

12 Houshian S, Schrøder HA, Weeth R. Correction of Madelung's deformity by the Ilizarov technique. J Bone Joint Surg Br 2004;86 (04):536-540

13 McCarroll HR Jr, James MA, Newmeyer WL III, Molitor F, Manske PR. Madelung's deformity: quantitative assessment of x-ray deformity. J Hand Surg Am 2005;30(06):1211-1220

14 McCarroll HR Jr,, James MA, Newmeyer WL 3rd, Manske PR. Madelung's deformity: diagnostic thresholds of radiographic measurements. J Hand Surg Am. 2010;35(05):807-12

15 Paes EC, Theunissen CMJ, Sakkers RJB, Schuurman AH. Langenskiöld procedure for Madelung's deformity: case series of late sequelae. Open J Orthop 2014;4:313-320
16 Steinman S, Oishi S, Mills J, Bush P, Wheeler L, Ezaki M. Volar ligament release and distal radial dome osteotomy for the correction of Madelung deformity: long-term follow-up. J Bone Joint Surg Am 2013;95(13):1198-1204

17 El-Gafary K, El-adly W. Forearm lengthening using Ilizarov external fixator. Eur Orthop Traumatol 2013;4:217-224

18 Mallard F, Jeudy J, Rabarin F, et al. Reverse wedge osteotomy of the distal radius in Madelung's deformity. Orthop Traumatol Surg Res 2013;99(4, Suppl):S279-S283

19 Imai Y, Miyake J, Okada K, Murase T, Yoshikawa H, Moritomo H. Cylindrical corrective osteotomy for Madelung deformity using a computer simulation: case report. J Hand Surg Am 2013;38(10): 1925-1932

20 Peymani A, Johnson AR, Dowlatshahi AS, et al. Surgical management of Madelung deformity: a systematic review. Hand (N Y) 2018;1558944718793179

21 Melhem E, Assi A, El Rachkidi R, Ghanem I. EOS(®) biplanar X-ray imaging: concept, developments, benefits, and limitations. J Child Orthop 2016;10(01):1-14

22 Dubousset J, Charpak G, Dorion I, et al. [A new 2D and 3D imaging approach to musculoskeletal physiology and pathology with low-dose radiation and the standing position: the EOS system]. Bull Acad Natl Med 2005;189(02):287-297, discussion 297-300

23 Chaibi Y, Cresson T, Aubert B, et al. Fast 3D reconstruction of the lower limb using a parametric model and statistical inferences and clinical measurements calculation from biplanar X-rays. Comput Methods Biomech Biomed Engin 2012;15 (05):457-466

24 Folinais D, Thelen P, Delin C, Radier C, Catonne Y, Lazennec JY. Measuring femoral and rotational alignment: EOS system versus computed tomography. Orthop Traumatol Surg Res 2013;99(05): 509-516

25 Escott BG, Ravi B, Weathermon AC, et al. EOS low-dose radiography: a reliable and accurate upright assessment of lower-limb lengths. J Bone Joint Surg Am 2013;95(23):e1831-e1837

26 Kim JW, Lee Y, Seo J, et al. Clinical experience with threedimensional printing techniques in orthopedic trauma. J Orthop Sci 2018;23(02):383-388

27 Zheng W, Chen C, Zhang C, Tao Z, Cai L. The feasibility of 3D printing technology on the treatment of pilon fracture and its effect on doctor-patient communication. BioMed Research International 2018:8054698

28 Zheng W, Su J, Cai L, et al. Application of 3D-printing technology in the treatment of humeral intercondylar fractures. Orthop Traumatol Surg Res 2018;104(01):83-88

29 Zheng W, Tao Z, Lou Y, et al. Comparison of the conventional surgery and the surgery assisted by 3D printing technology in the treatment of calcaneal fractures. J Invest Surg 2017 $1-11$

30 Sternheim A, Daly M, Qiu J, et al. Navigated pelvic osteotomy and tumor resection: a study assessing the accuracy and reproducibility of resection planes in Sawbones and cadavers. J Bone Joint Surg Am 2015;97(01):40-46

31 Iorio R, Pagnottelli $M$, Vadalà $A$, et al. Open-wedge high tibial osteotomy: comparison between manual and computer-assisted techniques. Knee Surg Sports Traumatol Arthrosc 2013;21(01): 113-119 